\title{
Melatonin synergized with cyclosporine A improves cardiac allograft survival by suppressing inflammation and apoptosis
}

\author{
CHEN LIU, TAO HONG, MENGPING SHAO, ZHENGHANG CHEN and CHUNSHENG WANG \\ Department of Cardiac Surgery, Zhongshan Hospital, Fudan University, Shanghai 200032, P.R. China
}

Received December 27, 2013; Accepted February 5, 2014

DOI: $10.3892 / \mathrm{mmr} .2014 .2382$

\begin{abstract}
Melatonin, a widespread physiological mediator, has been demonstrated to exhibit a dose-dependent immunoregulatory effect in vitro and in vivo, including mediating physiological circadian rhythms, neutralizing free radicals and exerting antisenescence actions. In the present study, the efficacy and mechanism of melatonin alone or in combination with cyclosporine (CsA) in prolonging heart transplantation survival was examined. Daily treatment with melatonin $(200 \mathrm{mg} / \mathrm{kg} /$ day $)$ through a gavage, significantly prolonged the survival of cardiac grafts (mean survival time, $13.4 \pm 2.4$ days; $\mathrm{n}=7 ; \mathrm{P}<0.0001)$ compared with the untreated controls $(5.8 \pm 1.2$ days; $\mathrm{n}=7)$. When CsA $(5 \mathrm{mg} / \mathrm{kg} /$ day) was co-administered with melatonin $(50 \mathrm{mg} / \mathrm{kg} / \mathrm{day})$, the survival rate improved (31.6 2.4 days; $\mathrm{n}=7$; $\mathrm{P}<0.001)$, compared with that achieved by only $20 \mathrm{mg} / \mathrm{kg} /$ day CsA ( $22 \pm 2.8$ days; $\mathrm{n}=7$ ). As expected, melatonin significantly alleviated the inflammatory response and apoptosis as determined by TdT-mediated dUTP-biotin nick end labeling assay $(\mathrm{P}<0.05)$. Further analysis demonstrated that melatonin significantly reduced $\mathrm{p} 65$ activation and the release of inflammatory factors. Therefore, these findings indicate that melatonin in combination with CsA protected the cardiac allograft by inhibiting inflammation-induced apoptosis. These results provide evidence for a novel therapeutic approach for future immunosuppressive agents in organ transplantation.
\end{abstract}

\section{Introduction}

Cardiac transplant is a useful therapeutic tool for the treatment of patients with end-stage heart failure or severe coronary artery disease (1). Despite marked effort and advances made during the past few years, with improved immunosuppression regimens and post-operative care, there are a number of

Correspondence to: Professor Chunsheng Wang, Department of Cardiac Surgery, Zhongshan Hospital, Fudan University, 180 Fenglin Road, Shanghai 200032, P.R. China

E-mail: liu.chen@zs-hospital.sh.cn

Key words: melatonin, cardiac allograft, cyclosporine A, proinflammatory cytokines, apoptosis problems that remain to be resolved, including poor long-term cardiac allograft outcomes, infections and malignancies (2-4). The introduction of cyclosporine (CsA) in immunosuppressive therapy has greatly improved the clinical outcome of cardiac transplantation $(5,6)$. However, the side effects of CsA, including irreversible pulmonary hypertension, infection and cancer, are serious limitations due to the high dosage required $(7,8)$. More recent strategies have focused on using cell therapies from different sources to facilitate stimulating the regeneration of cells inside the transplanted heart. However, the effect of differentiation on the expression profile of MHC proteins in allogeneic cell therapies is largely unknown (9). The development of a novel, short-term and effective immunomodulatory strategy is urgently required.

Melatonin, secreted by the pineal gland, is a widespread physiological mediator (10). Melatonin is a multifunctional gene, affecting various systems, including regulating circadian rhythm, immunoregulation, anti-oxidative effect and infection (11). Early evidence indicated that melatonin regulates the expression of Th1/2 cytokines, including tumor necrosis factor- $\alpha$ (TNF- $\alpha$ ), $\gamma$-interferon, interleukin (IL)-12, IL-1, IL-2 and macrophage colony-stimulating factor $(11,12)$. Rats supplemented with high $(50 \mathrm{mg} / \mathrm{kg})$ doses of melatonin exhibited significantly elevated survival times due to the abrogation of alloimmune responses (13). Further investigation has demonstrated that immunosuppressive maintenance therapy with CsA and rapamycin affected endogenous melatonin secretion (14). Melatonin has exhibited a protective action against CsA-induced oxidative stress, nephrotoxic effects and autophagy in vivo or in vitro (15-17). Previous studies have demonstrated that high-dose melatonin treatment prolonged cardiac allograft survival without side effects (13). However, the combined effect of melatonin plus CsA remains to be elucidated and the molecular regulatory mechanisms are unclear.

In the present study, it was determined whether co-treatment with melatonin and CsA was able to improve the survival of cardiac allografts. In addition, the possible mechanisms underlying this effect were investigated.

\section{Materials and methods}

Animal care. Specific pathogen-free male Sprague-Dawley (SD) rats and Wistar rats, weighing 200-250 g, were obtained from Shanghai Slaccas Laboratory Animal Company Ltd. (Shanghai, China). The animals were housed in standard 
polypropylene cages (three rats per cage) in a constant temperature $\left(22 \pm 2^{\circ} \mathrm{C}\right)$ with a 12 -h light and dark cycle. The care of the animals and experimental protocols was approved by the Animal Care and Use Committee of Fudan College of Science (Shanghai, China).

Animal experiments. Inbred SD rats and inbred Wistar rats were used as the recipients and donors, respectively. A total of 40 allogenic heart-transplanted SD rats were randomly divided into four groups of ten. Donor heart grafts were transplanted into the abdominal cavity of SD recipients using standard microsurgical techniques under sterile conditions. The saline group and the other three groups were administered $200 \mathrm{mg} / \mathrm{kg} /$ day melatonin (Sigma, St. Louis, MO, USA), $20 \mathrm{mg} / \mathrm{kg} /$ day $\mathrm{CsA}$ and $50 \mathrm{mg} / \mathrm{kg} /$ day melatonin with $5 \mathrm{mg} / \mathrm{kg} /$ day $\mathrm{CsA}$, respectively. The rate and strength of allograft pulsations were determined each day to check the survival times. Rejection was defined as heartbeat cessation for one day.

TdT-mediated dUTP-biotin nick end labeling (TUNEL) assay. For the detection of DNA strand breaks, paraffin-embedded sections were stained with the in situ cell death detection kit (Promega Corporation, Madison, WI, USA) according to the manufacturer's instructions. Briefly, formalin-fixed sections were dehydrated in alcohol and defatted in xylenes. Following being permeabilized in proteinase $\mathrm{K}$ for $10 \mathrm{~min}$, endogenous peroxidase was deactivated by $0.3 \%$ hydrogen peroxide. Next, the sections were incubated with $10 \mu$ l TUNEL reaction mixture, containing terminal deoxynucleotidyl transferase and fluorescein isothiocyanate dUTP, for $60 \mathrm{~min}$ at $37^{\circ} \mathrm{C}$. These sections were then stained with 3, 3'-diaminobenzidine, following hematoxylin post-staining, and observed under an optical microscope (4XC-MS; Shanghai Lunjie Mechanical and Electronical Instrument Co., Ltd., Shanghai, China). TUNEL-positive cells were counted in twelve randomly selected fields from each slide at a magnification of $\mathrm{x} 200$. The percentage of TUNEL-positive cells was analyzed in ten cardiac sections from ten different rats.

Immunohistochemistry. All of the fresh tissues were fixed in $10 \%$ neutral buffered formalin and embedded in paraffin. A total of 5- $\mu \mathrm{m}$ paraffin-embedded sections were cut on a microtome and stained with hematoxylin and eosin (H\&E) and Masson's trichrome according to the standard procedures. Generally, the $5-\mu \mathrm{m}$ paraffin-embedded sections were de-paraffinized in a series of xylene and hydrated in a graded series of ethanol. The sections were washed briefly in distilled water and stained in hematoxylin solution for $10 \mathrm{~min}$. Histological specimens were evaluated by the International Society of Heart and Lung Tranplantation (ISHLT) grading system (18) with an optical microscope (4XC-MS; Shanghai Lunjie Mechanical and Electronical Instrument Co., Ltd.).

IL-2 and TNF- $\alpha$ determination. Serum was prepared from the blood collected five days following heart transplantation. The levels of IL-2 and TNF- $\alpha$ were measured in the plasma acquired from the ELISA assay, which was stimulated with saline, melatonin, CsA or melatonin with CsA. IL-2 ELISAs were performed using the reagents and instructions provided by the manufacturer (Rat IL-2 Quantikine ELISA kit; R\&D Systems Inc., Minneapolis, MN, USA). TNF- $\alpha$ ELISAs were performed using the reagents and instructions provided by the manufacturer (Rat TNF- $\alpha$ Quantikine ELISA kit; R\&D Systems Inc.). All of the plasma specimens were examined in duplicate and expressed in $\mathrm{pg} / \mathrm{ml}$.

Western blot analysis. The lysis of the frozen left ventricle tissue samples were harvested in RIPA lysis buffer with proteinase inhibitor (Roche Diagnostics, Ltd., Lewes, UK), sonicated for $30 \mathrm{sec}$ and finally centrifuged for $30 \mathrm{~min}$ at $16,200 \mathrm{x} \mathrm{g}$. The proteins ( $\sim 60 \mu \mathrm{g}$ protein) from the total tissue samples were resolved by the $10 \%$ sodium dodecyl sulfate-polyacrylamide gel electrophoresis and then transferred to the pure nitrocellulose membrane (Bio-Rad, Hercules, CA, USA). Blocking was performed with $5 \%$ non-fat dry milk overnight at $4{ }^{\circ} \mathrm{C}$ and incubated with mouse monoclonal Bcl-2 antibody (Proteintech Group Inc., Wuhan, China; cat no. 60178-1-Ig), mouse monoclonal IL-1 $\beta$ antibody (Cell Signaling Technology, Inc., Beverly, MA, USA; cat no. 8689), rabbit polyclonal Phospho-NF-кB p65 (Ser536) antibody (Cell Signaling Technology, Inc.; cat no. 3031), rabbit polyclonal NF-кB p65 (C22B4; Cell Signaling Technology, Inc.; cat no. 4764) and rabbit polyclonal $\beta$-actin (Sigma; cat no. A2066) overnight at $4^{\circ} \mathrm{C}$. The western blots were processed using a horseradish peroxidase goat anti-rabbit IgG (Shanghai Kangcheng Bioengineering Co., Ltd., Shanghai, China). The Pierce enhanced chemiluminescence Western Blotting Substrate was used for chemiluminescence detection according to the manufacturer's instructions (Pierce Biotechnology, Inc., Rockford, IL, USA). Densitometric analysis of protein was conducted using Bio-Rad Quantity One ${ }^{\circledR}$ 1-D software (Bio-Rad).

Statistical analysis. Each experiment was performed at least three times and the representative data were collected. Data are presented as the mean \pm standard deviation. Differences between the four groups were determined using one-way analysis of variance. The statistical analysis was performed using the GraphPad Prism V5.03 software (GraphPad, San Diego, CA, USA). $\mathrm{P}<0.05$ was considered to indicate a statistically significant difference.

\section{Results}

Melatonin with CsA markedly prolongs cardiac allograft survival. Previous studies have demonstrated that melatonin is able to suppress acute cardiac allograft rejection in vivo. The present study investigated the effects on acute cardiac allograft rejection of melatonin only, CsA only and melatonin with CsA. As revealed in Fig. 1, the survival times of hearts transplanted to animals treated with melatonin were significantly prolonged compared with the untreated control in a rat cardiac allograft model starting at the day of transplantation. However its efficacy was less than that of $20 \mathrm{mg} / \mathrm{kg}$ CsA. By contrast, $50 \mathrm{mg} / \mathrm{kg}$ melatonin in combination with $5 \mathrm{mg} / \mathrm{kg}$ CsA was more effective than the sole administration of $20 \mathrm{mg} / \mathrm{kg} \mathrm{CsA}$ (Fig. 1). These data indicated that melatonin exhibits a beneficial effect on allograft survival and may offer the possibility of reducing the dose of CsA used in patients, thereby limiting the potential for severe side effects. 

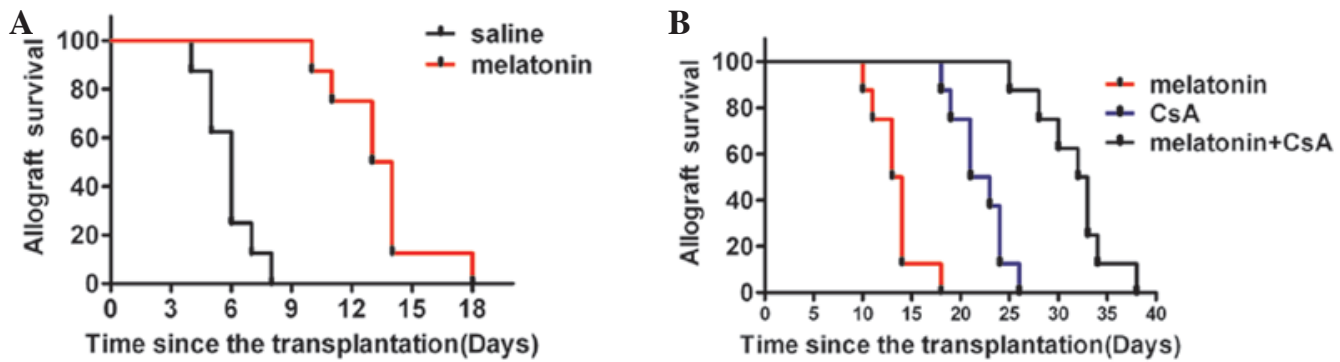

Figure 1. Melatonin significantly improved allograft survival. Graft survival of Wistar cardiac grafts were transplanted heterotopically to the abdominal vessels of Sprague-Dawley rat recipients in various treatment cohorts following transplantation. (A) Long term graft survival was significantly greater with melatonin (mean survival, 13.4 \pm 2.4 days) compared with the untreated control (5.8 \pm 1.2 days, $n=10)$. (B) Melatonin synergied with CsA markedly prolonged heart allograft survival $(31.6 \pm 2.4$ days; $\mathrm{n}=10 ; \mathrm{P}<0.001)$. CsA, cyclosporine.

A

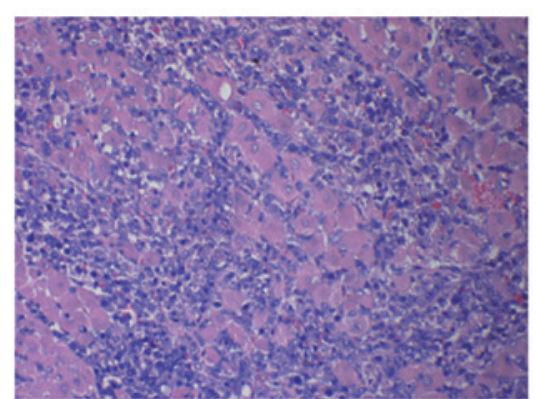

C

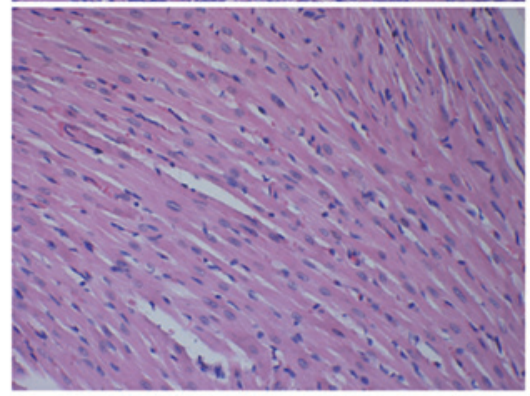

$\mathbf{E}$

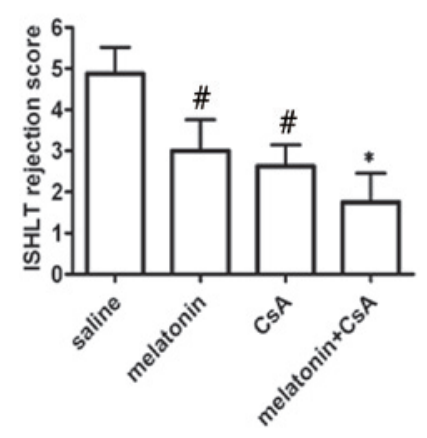

$\mathbf{F}$

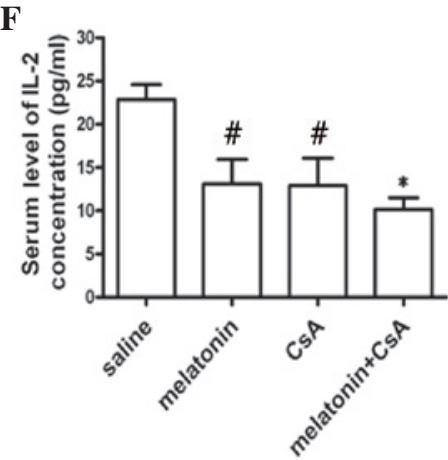

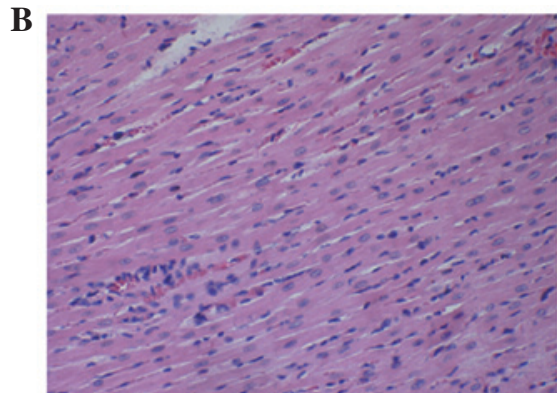

D

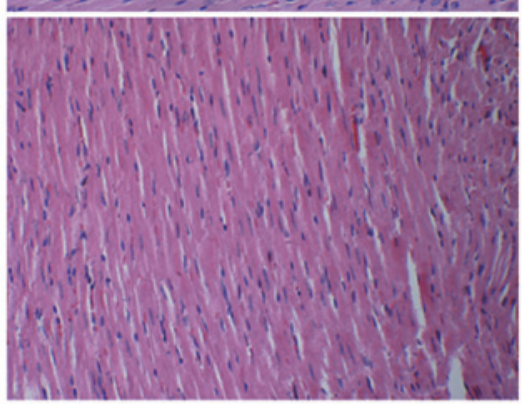

G

Figure 2. Assessment of local immune rejection by H\&E staining. Left ventricles were collected five days following transplantation. Representative images of cardiac histology from rats treated with (A) saline as the control, (B) melatonin alone, (C) CsA alone and (D) combination of melatonin and CsA are shown (stain, H\&E; magnification, x100). (E) ISHLT rejection scores in acute cardiac rejection in different groups following transplantation. (F and G) Serum was collected five days following graft. Serum IL-2 and TNF- $\alpha$ were measured. All data were expressed as the mean \pm standard deviation ( $=10$ ). ${ }^{\#} \mathrm{P}<0.05$ as compared with the control; * $\mathrm{P}<0.05$ compared with melatonin or CsA group. CsA, cyclosporine; H\&E, hematoxylin and eosin; ISHLT, International Society for Heart and Lung Transplantation; IL-2, interleukin-2; TNF- $\alpha$; tumor necrosis factor- $\alpha$.

Melatonin suppresses inflammatory responses in vivo. Imbalances in inflammatory processes are important in the pathogenesis of immune rejection. In order to examine acute immune rejection in the myocardium, H\&E-stained sections were evaluated using the ISHLT grading system (18). As demonstrated in Fig. 2B and C, the cardiac histopathology revealed that $50 \mathrm{mg} / \mathrm{kg}$ melatonin and $20 \mathrm{mg} / \mathrm{kg}$ CsA was able to extenuate the inflammatory reaction. While $50 \mathrm{mg} / \mathrm{kg}$ melatonin co-administrated with $5 \mathrm{mg} / \mathrm{kg}$ CsA significantly alleviated heart congestion (Fig. 2D). The rejection grade diagnosed by ISHLT confirmed the histological results (Fig. 2E). Further analysis revealed that treatment with melatonin significantly attenuated the release of inflammatory cytokines, including IL-2 and TNF- $\alpha$ (Fig. 2 F and G). These 
A

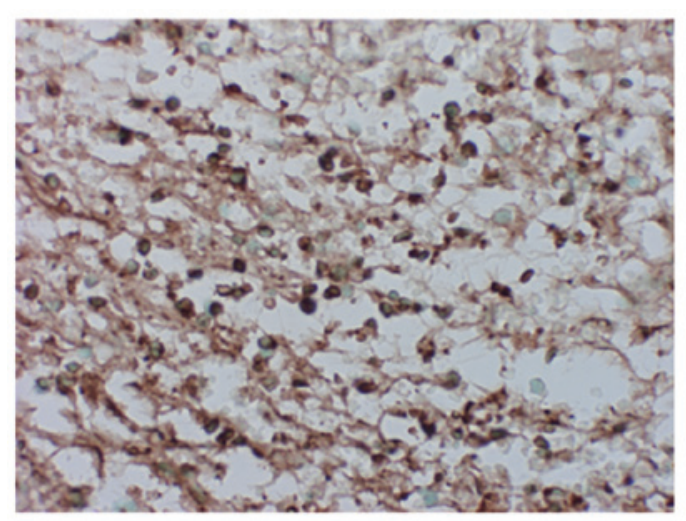

C

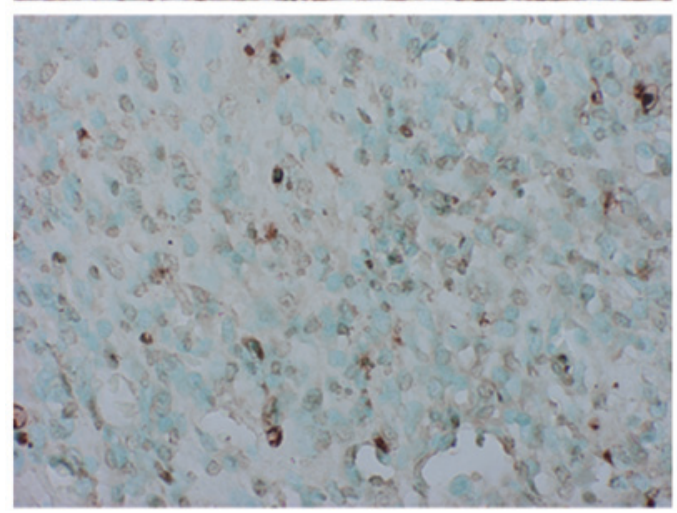

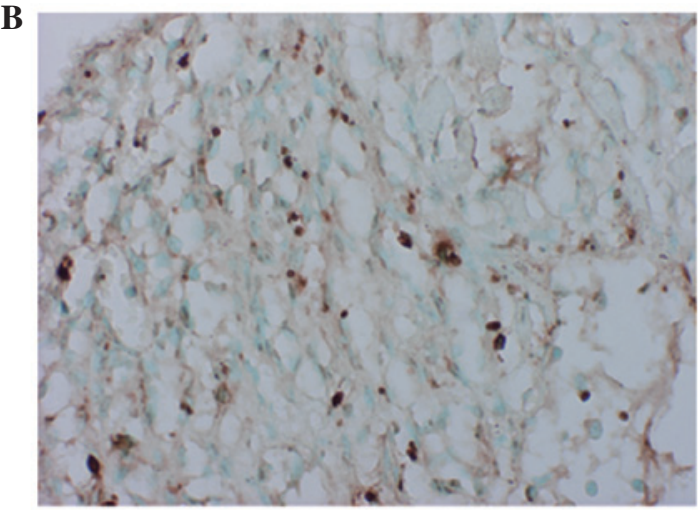

D

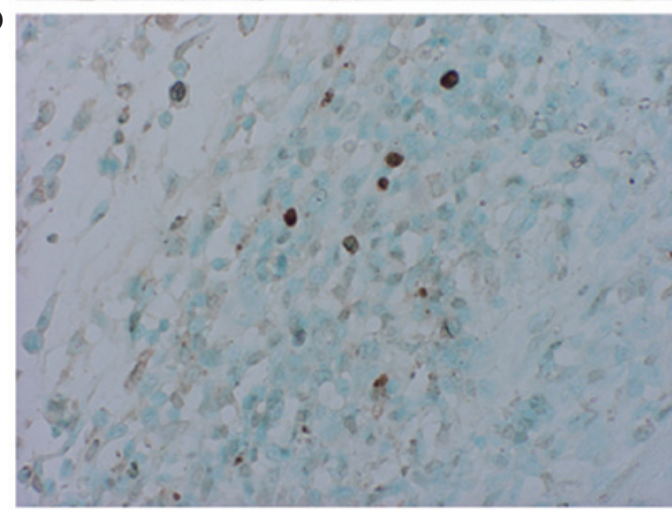

$\mathbf{E}$

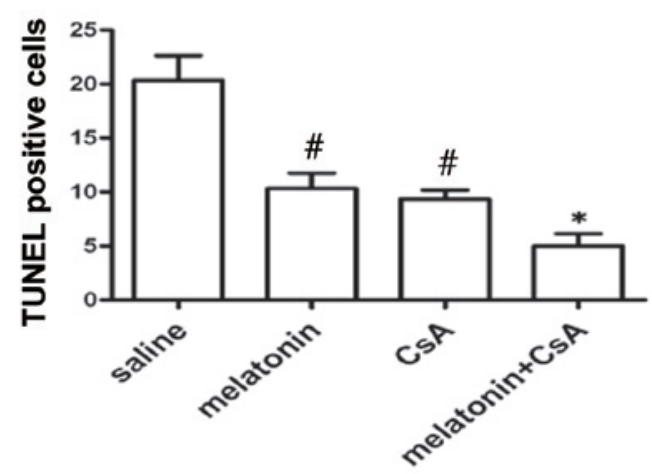

Figure 3. Melatonin protects against apoptosis. Cardiac samples $(\mathrm{n}=3)$ were collected on five days following transplantation. Myocardium death was determined by a TUNEL assay. Representative photomicrographs of left ventricles from rats treated with A) saline as the control, (B) melatonin alone, (C) CsA alone and (D) combination of melatonin and CsA are demonstrated. (magnification, x100; stained using a TUNEL kit). (E) Densitometry analysis of immunoblots for the ratio of TUNEL-positive cells in each group. All data are expressed as the mean \pm standard deviation $(\mathrm{n}=10)$. ${ }^{\#} \mathrm{P}<0.05$ as compared with the control. ${ }^{*} \mathrm{P}<0.05$, compared with the melatonin or CsA group. CsA, cyclosporine; TUNEL, TdT-mediated dUTP-biotin nick end labeling assay.

results suggested that melatonin attenuated immune rejection by reducing the activation of inflammatory responses.

Melatonin protects against apoptosis of cardiac myocytes. The number of apoptotic cardiac myocytes increased markedly in association with the increased infiltration of macrophages into the myocardial tissue during the course of cardiac allograft rejection. Melatonin has been reported to exhibit an antiapoptotic effect in other tissues. Thus, the present study investigated the role of melatonin on apoptosis in myocardial tissue on day five following transplantation. Apoptosis was observed in all myocardial tissue and the number of apoptotic cardiac myocytes significantly reduced in the allografts treated with $200 \mathrm{mg} / \mathrm{kg}$ melatonin or $20 \mathrm{mg} / \mathrm{kg} \mathrm{CsA}$, compared with in the allografts administered saline (Fig. 3A-C). When $50 \mathrm{mg} / \mathrm{kg}$ melatonin with less CsA $5 \mathrm{mg} / \mathrm{kg}$ was administered to the animals, the proportion of apoptotic cardiac myocytes was 6 -fold lower than the saline group and 2-fold lower than the rats treated with melatonin or CsA alone (Fig. 3D). These data suggested that melatonin significantly reduces the number of TUNEL-positive cells.

Expression levels of $p 65, \mathrm{Bcl}-2$ and $I L-1 \beta$ in left ventricular tissue. To examine the possible mechanisms underlying the improvement of allograft survival in melatonin-treated rats, the present study focused on the expression of p65, Bcl-2 and IL-1 $\beta$, which are key genes in inflammation and apoptosis. As expected, it was identified that the expression of p-p65 (Ser536) also significantly decreased in the $200 \mathrm{mg} / \mathrm{kg}$ melatonin or $20 \mathrm{mg} / \mathrm{kg}$ CsA-treated rats in comparison with the saline-treated animals (Fig. 4A). Consistent with p-p65, the expression of IL-1 $\beta$ was also reduced and the level of Bcl-2 


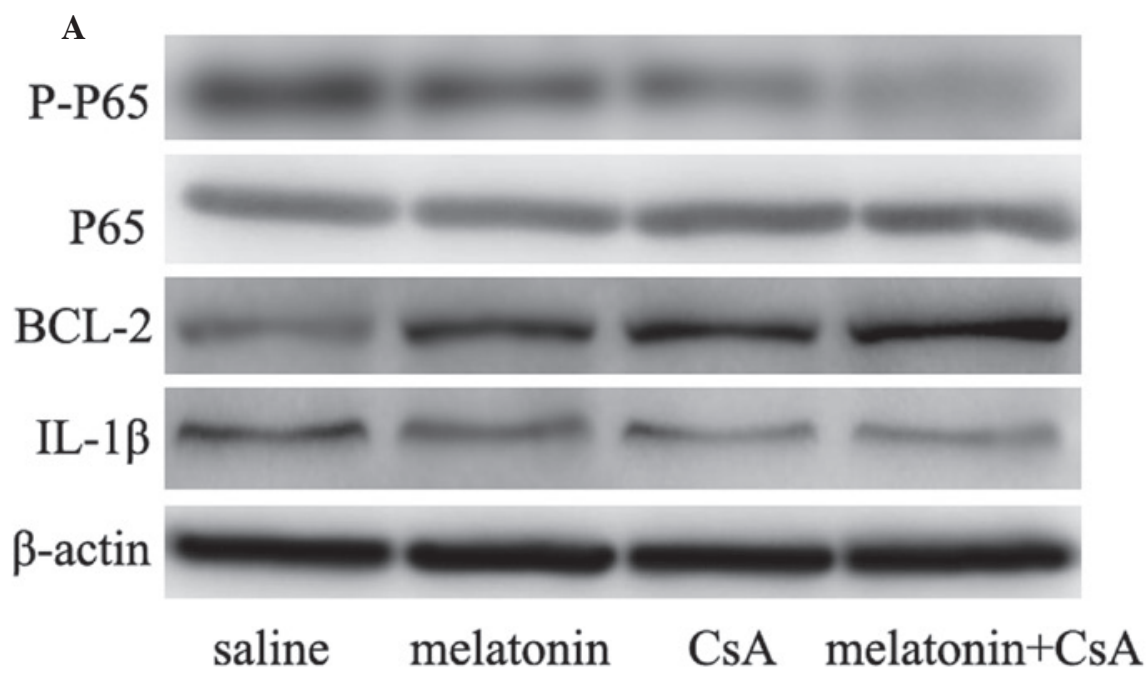

B

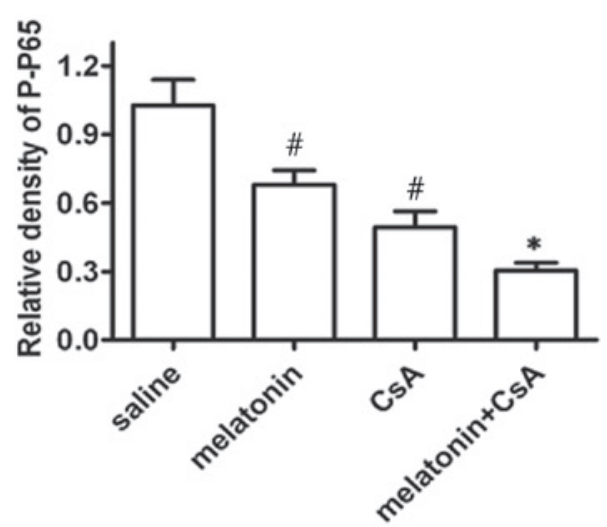

D

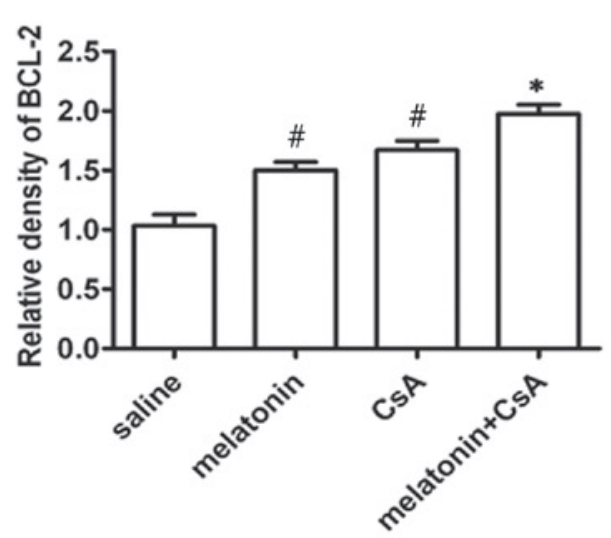

C

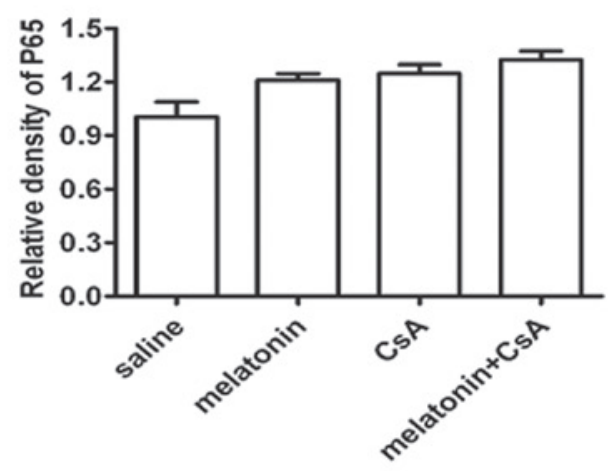

$\mathbf{E}$

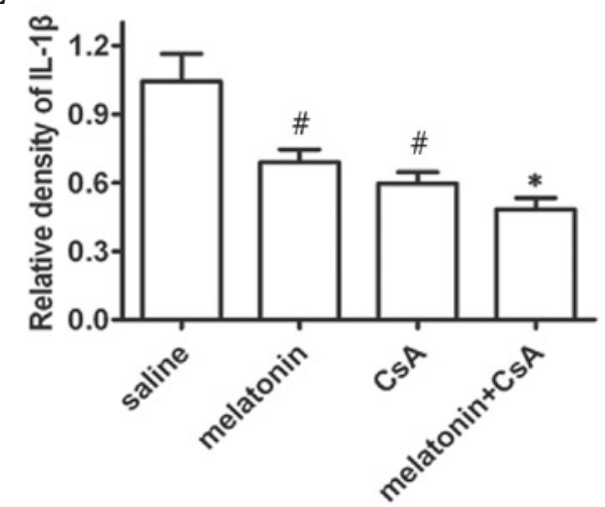

Figure 4. Western blot analysis the expression of p65, Bcl-2 and IL-1 $\beta$. (A) Immunoblots were conducted to determine the protein levels of p65, Bcl-2 and IL-1 $\beta$, and the protein expression levels were normalized to $\beta$-actin values. (B) Density levels of p-p65, (C) p65, (D) Bcl-2 and (E) IL-1 $\beta$ were quantified using the Quantity One ${ }^{\circledR}$ program. Data represent the means of three separate experiments. ${ }^{\#} \mathrm{P}<0.05$ as compared with the control; ${ }^{*} \mathrm{P}<0.05$ compared with the melatonin or CsA group. CsA, cyclosporine; IL- $\beta$, interleukin- $\beta$.

was upregulated (Fig. 4). Notably, $50 \mathrm{mg} / \mathrm{kg}$ melatonin with $5 \mathrm{mg} / \mathrm{kg}$ CsA have an effective inhibition on the expression of p-p65 (Ser536) and IL-1 $\beta$ (Fig. 4). These data indicated that melatonin may inhibit immune rejection to reduce the release of inflammatory cytokines that induce apoptosis.

\section{Discussion}

In the present study, it was demonstrated that allograft viability is prolonged by direct modulation of host immunity via concomitant immunosuppression with melatonin-treatment by utilizing a well-characterized experimental rat cardiac transplantation model. Melatonin ameliorated prolonged inflammation and the associated release of proinflammatory cytokines and chemokines, which lead to transplant pathology. Serum levels of TNF- $\alpha$ and IL-2 were significantly reduced in vitro in the presence of melatonin and/or CsA, which may contribute to enhanced cardiac allograft survival.

These data in a heart transplant model suggested that melatonin effectively prolongs the survival of cardiac allografts 
and reduces the dose of CsA. CsA has a marked beneficial effect in organ transplantation in vivo and in vitro, however, there are a number of significant side effects. In the present study, the rats were treated with melatonin and CsA, which effectively suppressed the activity of the adaptive immune response, including the release of IL- 2 and TNF- $\alpha$ in serum, which was consistent with previous studies $(13,19)$. The protein levels of p-p65 and IL-1 $\beta$ also markedly declined. Several studies have demonstrated that melatonin increases the production of TNF- $\alpha$ (20). The discrepancy may be as a result of the dose of melatonin or the experimental model used.

Apoptosis is the process of programmed cell death distinct from necrosis $(21,22)$. Apoptotic myocytes are mainly localized adjacent to areas of inflammatory infiltration $(23,24)$, so the coercion of apoptosis protects the cardiac myocytes. The results indicated that melatonin was able to reduce the number of apoptotic myocytes and the upregulation of $\mathrm{Bcl}-2$. The combination of melatonin and CsA had an additive effect, which provided an effective approach to alleviate the proinflammatory state associated with acute graft rejection.

\section{Acknowledgements}

The study was supported by the National Natural Science Foundation of China (grant no 81270326).

\section{References}

1. Gill JS: Cardiovascular disease in transplant recipients: current and future treatment strategies. Clin J Am Soc Nephrol 3 (Suppl 2): S29-S37, 2008

2. Bruschi G, Colombo T, Oliva F, et al: Heart transplantation: 25 years' single-centre experience. J Cardiovasc Med (Hagerstown) 14: 637-647, 2013.

3. Fiorelli AI, Branco JN and Dinkhuysen JJ, et al: Risk factor analysis of late survival after heart transplantation according to donor profile: a multi-institutional retrospective study of 512 transplants. Transplant Proc 44: 2469-2472, 2012.

4. Suzuki J, Ogawa M, Hirata Y, Nagai R and Isobe M: Effects of immunoglobulin to prevent coronary allograft vasculopathy in heart transplantation. Expert Opin Ther Targets 16: 783-789, 2012.

5. Tedoriya T, MacDonald PS, Keogh AM, Wilson M and Spratt PM: Reversal of chronic cyclosporin nephrotoxicity after heart transplantation. J Heart Lung Transplant 20: 247-248, 2001.

6. Aumente MD, Arizón JM, Segura J, et al: Relationship between pharmacokinetic parameters of cyclosporin and the incidence of acute rejection after heart transplantation. Transplant Proc 37: 4014-4017, 2005.

7. Baan CC, Vaessen LM, Balk AH, et al: Cyclosporin A sensitivity of allo-specific precursor and committed cytotoxic T lymphocytes after clinical heart transplantation. Transplant Proc 26: 2849-2851, 1994.
8. Werkö L: Cyclosporin as a cause of hypertension in patients with heart transplantation. Lakartidningen 88: 503, 1991 (In Swedish).

9. Huang XP, Sun Z, Miyagi Y, et al: Differentiation of allogeneic mesenchymal stem cells induces immunogenicity and limits their long-term benefits for myocardial repair. Circulation 122: 2419-2429, 2010.

10. Espino J, Pariente JA and Rodríguez AB: Oxidative stress and immunosenescence: therapeutic effects of melatonin. Oxid Med Cell Longev 2012: 670294, 2012.

11. Fildes JE, Yonan N and Keevil BG: Melatonin - a pleiotropic molecule involved in pathophysiological processes following organ transplantation. Immunology 127: 443-449, 2009.

12. Gilad E, Wong HR, Zingarelli B, et al: Melatonin inhibits expression of the inducible isoform of nitric oxide synthase in murine macrophages: role of inhibition of NFkappaB activation. Faseb J 12: 685-693, 1998.

13. Jung FJ, Yang L, Härter L, et al: Melatonin in vivo prolongs cardiac allograft survival in rats. J Pineal Res 37: 36-41, 2004.

14. Cardell M, Jung FJ, Zhai W, et al: Acute allograft rejection and immunosuppression: influence on endogenous melatonin secretion. J Pineal Res 44: 261-266, 2008.

15. Eşrefoğlu M1, Kuruş M and Sahna E: The beneficial effect of melatonin on chronic cyclosporin A nephrotoxicity in rats. J Int Med Res 31: 42-44, 2003.

16. Rezzani R, Buffoli B, Rodella L, Stacchiotti A and Bianchi R: Protective role of melatonin in cyclosporine A-induced oxidative stress in rat liver. Int Immunopharmacol 5: 1397-1405, 2005.

17. Yoo YM and Jeung EB: Melatonin suppresses cyclosporine A-induced autophagy in rat pituitary GH3 cells. J Pineal Res 48: 204-211, 2010.

18. Jessup M, Banner $\mathrm{N}$ and Brozena $\mathrm{S}$, et al: Optimal pharmacologic and non-pharmacologic management of cardiac transplant candidates: approaches to be considered prior to transplant evaluation: International Society for Heart and Lung Transplantation guidelines for the care of cardiac transplant candidates - 2006. J Heart Lung Transplant 25: 1003-1023, 2006.

19. Carrillo-Vico A, Lardone PJ, Naji L, et al: Beneficial pleiotropic actions of melatonin in an experimental model of septic shock in mice: regulation of pro-/anti-inflammatory cytokine network, protection against oxidative damage and anti-apoptotic effects. J Pineal Res 39: 400-408, 2005.

20. Santello FH, Frare EO, Caetano LC, AlonsoToldo MP and do Prado JC Jr: Melatonin enhances pro-inflammatory cytokine levels and protects against Chagas disease. J Pineal Res 45: 79-85, 2008

21. Tang EH, Libby P, Vanhoutte PM and Xu A: Anti-inflammation therapy by activation of prostaglandin EP4 receptor in cardiovascular and other inflammatory diseases. J Cardiovasc Pharmacol 59: 116-123, 2012.

22. Wolters SL, Corsten MF, Reutelingsperger CP, Narula J and Hofstra L: Cardiovascular molecular imaging of apoptosis. Eur J Nucl Med Mol Imaging 34 (Suppl 1): S86-S98, 2007.

23. Szabolcs M, Michler RE and Yang X, et al: Apoptosis of cardiac myocytes during cardiac allograft rejection. Relation to induction of nitric oxide synthase. Circulation 94: 1665-1673, 1996.

24. Gedik HS, Korkmaz K, Erdem H, Karakilic E, Lafci G and Ankarali $\mathrm{H}$ : Protective effect of heparin in the end organ ischemia/reperfusion injury of the lungs and heart. J Cardiothorac Surg 7: 123, 2012. 